\title{
Heterogeneity in functional status among moderately frail older adults: improving predictive performance using a modified approach of subgrouping the Clinical Frailty Scale
}

\author{
Edward Chong ${ }^{1,2}$ (D) Mark Chan ${ }^{1,2} \cdot$ Huei Nuo $\operatorname{Tan}^{1,2} \cdot$ Wee Shiong Lim $^{1,2}$
}

Received: 22 July 2020 / Accepted: 8 October 2020 / Published online: 23 October 2020

(c) European Geriatric Medicine Society 2020

\section{Key summary points}

Aims To establish if dependency in basic activities of daily living (bADL) amongst moderately frail older adults predict poorer health outcomes including mortality and institutionalisation. We also examined the utility of subgrouping category 6 of the Clinical Frailty Scale (CFS) by level of functional dependency to improve predictive performance.

Findings We observed a wider range in functional dependency among CFS 6 patients when compared to other frail categories. Incorporating CFS 6 subcategories based on bADL functional status increased predictive performance for longitudinal adverse outcomes compared with the original CFS scoring.

Message This study corroborates the heterogeneity of bADL functional status in CFS 6 individuals and validates the use of a modified approach to subgrouping the CFS via bADL dependency for improved predictive performance.

\begin{abstract}
Purpose Moderately frail individuals [Clinical Frailty Scale (CFS) 6] demonstrate heterogeneity in basic activities of daily living (bADL). We aimed to establish whether functional dependency in moderate frailty predicts poorer outcomes and examined the utility of subgrouping the CFS in predicting mortality and institutionalisation.

Methods We prospectively studied 201 hospitalised frail patients $(89.5 \pm 4.7$ years, female $70.1 \%)$. We examined Katz Index (KI) against adverse outcomes in CFS6 $(n=106)$. We then compared predictive performances of a modified CFS version 1 (mCFS-1; category 6A: CFS6 and $\mathrm{KI} \geq 2$; 6B: CFS6 and $\mathrm{KI} \leq 1$ ) and modified CFS version 2 (mCFS-2; category 6A: CFS6 and $\mathrm{KI} \geq 2$; 6B1: $\mathrm{CFS} 6, \mathrm{KI} \leq 1$ and feeding independent; 6B2: $\mathrm{CFS} 6, \mathrm{KI} \leq 1$ and feeding dependent) against the CFS. Multivariate analysis was used to compare each tool against mortality and institutionalisation. Receiver operator characteristic analysis was performed to determine area under curve and optimal cut-points for each tool.

Results $\mathrm{KI} \leq 1$ in CFS6 was associated with higher 12 -month mortality (39.3\% vs. $15.6 \%, p=0.01$ ); amongst KI items, feeding dependent predicted 12 -month mortality $(p<0.05)$. Using mCFS-1, category 6A did not increase 12-month mortality compared with category 5 (OR 1.83, 95\% CI 0.52-6.47), unlike category 6B (OR 6.33, 95\% CI 2.07-19.33). mCFS-2 produced higher mortality in category 6B1 (OR 5.19, 95\% CI 1.30-20.69) and 6B2 (OR 6.92, 95\% CI 2.14-22.35). Similar observations were seen for institutionalisation. Optimal cut-point for 12-month mortality was category 6 for CFS, and 6B and 6B1 for mCFS-1 and mCFS-2, respectively.
\end{abstract}

Electronic supplementary material The online version of this article (https://doi.org/10.1007/s41999-020-00418-8) contains supplementary material, which is available to authorized users.

Edward Chong

edward_chong@ttsh.com.sg

1 Department of Geriatric Medicine, Tan Tock Seng Hospital, 11 Jalan Tan Tock Seng, Singapore 308433, Singapore

2 Institute of Geriatrics and Active Ageing, Tan Tock Seng Hospital, Singapore, Singapore 
Conclusion This study corroborates the heterogeneity of functional status in moderately frail individuals and validates the use of a modified approach to subgrouping the CFS6 via bADL functional status for improved predictive performance.

Keywords Geriatrics $\cdot$ Frailty $\cdot$ Acute $\cdot$ Risks $\cdot$ Outcomes $\cdot$ Inpatient

\section{Introduction}

Frailty is a multidimensional syndrome of reduced physiological functioning characterised by increased vulnerability to a myriad of adverse health outcomes, even when subjected to minor stressors [1]. Over the years, frailty has become an increasingly integral part of clinical practice and healthcare delivery $[2,3]$. There are many tools which have been developed for frailty screening and identification. Amidst the array of frailty tools, the Clinical Frailty Scale (CFS) stands out as a simple-to-use, validated and reliable tool for frailty assessment [4-8].

Although the CFS was initially designed as a global synthesis assessment tool, it has rapidly evolved into an effective screening tool across various healthcare settings [2, 9]. The 9-point scale allows classification across the frailty continuum ranging from 1 (very fit) to 9 (terminally ill) with each category having a brief description and visual depiction. Frailty is diagnosed when individuals are categorised as CFS 5-8. The CFS is also a well-validated predictive tool for adverse health outcomes in older adults including mortality [4-7], institutionalisation [5-7], length of hospitalisation [10], risk of re-hospitalisation [10,11], and peri-operative complications [12]. Given its widespread use, the tool has also been validated for administration through various methods including telephonic interviews [13], retrospective medical chart review [14], and via standardised algorithm [7].

Although there remains a paucity of data, patients in CFS 6 (moderately frail) appear to demonstrate a wider variation in rating of level of dependency in basic activities of daily living (bADL). Whilst there are clear demarcations for difficulties with instrumental ADLs (CFS 5) and dependence in all aspects of bADL [CFS 7 (severely frail) and 8 (very severely frail)], CFS 6 encompasses a heterogeneous in-between group with varying degrees of dependency in bADL. This is not a moot point, as increasing functional dependency in bADL has been shown to be a strong independent predictor of negative health outcomes [15-17]. Hence, given the variation in bADL functional ability within CFS 6, the current practice of treating CFS 6 as a single entity in frailty assessment may be inadequate and nondiscriminatory in establishing risk estimates for negative health outcomes.

Against this backdrop, we performed a secondary analysis study of our original prospective study to determine if bADL functional ability (as represented by Katz Index total score and individual items) in moderately frail (CFS
6) older adults predicts outcomes of mortality and institutionalisation at initial hospitalisation and at 6- and 12-month follow-up. Using bADL functional ability to sub-categorise CFS 6, we studied how the expanded CFS 6 categories compared against mild (CFS 5) and severe (CFS 7-8) frailty in predicting mortality and institutionalisation. We also examined the predictive performance of the modified approach of subgrouping CFS 6 by bADL functional ability to predict outcomes compared with CFS.

\section{Methods}

\section{Study design and eligibility criteria}

We previously reported diagnostic and predictive performance of the CFS against the "gold-standard" Frailty Index $[5,6]$. We also validated a standardised approach in administration of the CFS using a simple algorithm (CFS-A) [7]. This study examined the same population of 210 older adults who were consecutively admitted in 2015 to the Department of Geriatric Medicine, Tan Tock Seng Hospital, Singapore. In addition to our original study's exclusion criteria, which were (1) previous recruitment in an earlier admission, (2) terminal illness (life expectancy less than a year), (3) patient identified as "dangerously ill" (typically a label used for patients who have hemodynamic instability, requiring close monitoring with or without the need for high dependency or critical care) for more than $72 \mathrm{~h}$ from day of admission, (4) absence of caregiver for consent in patients who lack mental capacity, and (5) current resident in a community hospital or nursing home, we further excluded individuals who were not frail. Hence, a total of 201 participants were included for analysis in this study as they were diagnosed as frail (CFS category 5-8) by two independent raters using the CFS-A approach [7].

Data on patient demographics, cognitive function, admitting diagnosis, functional status (Katz index), burden of comorbidities [modified Charlson's Comorbidity Index (CCI)] and severity of illness (modified severity of illness index) were gathered $[18,19]$. Further details of the original study methods have been described previously [5].

Written informed consent was obtained from the patient or a legally acceptable representative (in individuals who lack capacity for consenting). Ethics approval was obtained from the Domain Specific Review Board of the National Healthcare Group, Singapore. 


\section{Examining Katz Index (KI) and adverse health outcomes in CFS 6}

A total of 106 moderately frail (CFS 6) individuals were examined by comparing individual KI items (bathing, toileting, transfer, dressing, continence, and feeding) and total KI scores (0-6) against the outcomes of mortality and institutionalisation at initial hospitalisation, 6 and 12 months post enrolment. We then examined independent associations between each individual KI items against the aforementioned outcomes of interest.

\section{Modified approach of subgrouping the CFS 6 category}

Following subgroup analyses, and with the knowledge that feeding dependence and lower KI scores portend poorer outcomes, we propose two variations of a modified approach to subgroup CFS 6 (Appendix 1). Modified CFS version 1 (mCFS-1) involved separating CFS 6 into two sub-categories: (1) CFS 6A-category 6 with a total KI of 2-6, and (2) CFS 6B-category 6 with a total KI of $0-1$. Modified CFS version 2 (mCFS-2) involved separating CFS 6 into three sub-categories: (1) CFS 6A-category 6 with a total KI of 2-6, (2) CFS 6B1 - category 6 , total KI of $0-1$, and feeding independent, and (3) CFS 6B2-category 6 , total KI of $0-1$, and feeding dependent.

\section{Outcome measures}

All-cause mortality (as primary outcome) and institutionalisation were gathered via patients' electronic medical records and telephonically at initial hospitalisation, 6- and 12-month follow-up. Institutionalisation was captured as a composite outcome as participants who died during the specified period were counted as a case. This well-accepted method, which was performed in our original study [6,7], allows us to make the assumption that institutionalisation had occurred in all mortality cases. We also examined and compared CFS, mCFS-1, and mCFS- 2 in their predictive performance for the aforementioned outcomes of interest.

\section{Statistical analyses}

Data were captured using standardised data collection forms and entered into an electronic database. We expressed continuous variables as means (standard deviation) or medians (interquartile range), whilst categorical variables were expressed as counts and percentages. Univariate analysis was performed using Chi-square or Fisher Exact test for counts; Kruskall-Wallis test (for non-parametric data), and analysis of variance (ANOVA; for parametric data) were performed to compare baseline characteristics between three groups of frail patients-CFS 5, 6, and 7-8.

Focusing on category 6, we compared KI total and item scores against the outcomes of mortality and institutionalisation. We then performed logistic regression analysis, adjusting for age, gender, comorbidities, and severity of illness, to investigate the independent association between dependency in individual $\mathrm{KI}$ items and the adverse outcomes of interest.

Following subgroup analyses, we performed univariate analysis comparing the CFS, mCFS- 1 , and mCFS- 2 against mortality and institutionalisation. Logistic regression analyses, adjusting for age, gender, comorbidities, and severity of illness, were performed to compare predictive performances of each tool against mortality and institutionalisation. We also performed receiver operating curve analysis to determine area under curve (AUC) and optimal cut-point for each tool using Youden's Index $(J)$.

Statistical analysis was performed using SPSS V19.0 (SPSS, Inc., Chicago, IL, USA), and STATA V12.0 (Stata Corp, College Station, TX, USA) assuming a two-sided test at $5 \%$ level of significance.

\section{Results}

\section{Baseline characteristics}

A total of 201 participants were examined in this study. Of these, 60 (29.9\%), 106 (52.7\%), and 35 (17.4\%) patients were rated as CFS 5, 6, and 7-8, respectively. The mean age of our study participants was $89.5 \pm 4.7$ years, with predominance of females $(n=141,70.1 \%)$ and Chinese ethnicity $(n=164,81.6 \%)$ (Table 1$)$. Four participants were lost to follow-up resulting in a total of 197 patients for follow-up analyses.

Age, gender, ethnicity, and severity of illness were similar between CFS groups. We observed significantly higher comorbidities, dementia, and delirium as frailty severity increased. Total CCI score was significantly higher in CFS 6 [CCI 3.0 (2.0-4.0)] compared to CFS 5 [CCI $2.0(1.0-3.0)$ ] or CFS 7-8 [CCI $2.0(2.0-4.0)$ ] $(p<0.001)$.

We observed a wider range in functional dependency among CFS 6 patients [KI $1(0-3)$ ], when compared to CFS 5 [KI 6 (6-6)] and 7-8 [KI 0 (0-0)]. CFS 6 had the widest range of dependency in individual domains of KI, ranging from $40(37.7 \%)$ for feeding to 89 (84.0\%) for bathing.

\section{bADL dependency and adverse outcomes (CFS 6)}

Amongst 106 patients who are moderately frail (CFS 6), we observed a statistically significant association between dependency in feeding and mortality at 6 -month $(35.0 \%$ vs. 
Table 1 Baseline characteristics: comparison between CFS categories

\begin{tabular}{|c|c|c|c|c|}
\hline Baseline characteristics $(n=201)$ & CFS $5(n=60)$ & CFS $6(n=106)$ & CFS 7-8 $(n=35)$ & $p$ value \\
\hline Age $($ mean $\pm \mathrm{SD})$ & $88.5 \pm 4.3$ & $89.8 \pm 4.5$ & $90.1 \pm 5.4$ & 0.155 \\
\hline Race, Chinese (\%) & $55(91.7)$ & $82(77.4)$ & $27(77.1)$ & 0.309 \\
\hline Gender, Male (\%) & $20(33.3)$ & $30(28.3)$ & $10(28.6)$ & 0.780 \\
\hline \multicolumn{5}{|l|}{ Comorbidities } \\
\hline CCI (median, IQR) & $2(1-3)$ & $3(2-4)$ & $2(2-4)$ & 0.025 \\
\hline \multicolumn{5}{|l|}{ CCI class $(\%)$} \\
\hline Low & $6(10.0)$ & $9(8.5)$ & $0(0.0)$ & \multirow[t]{4}{*}{0.079} \\
\hline Medium & $30(50.0)$ & $38(35.8)$ & $20(57.1)$ & \\
\hline High & $20(33.3)$ & $41(38.7)$ & $9(25.7)$ & \\
\hline Very high & $4(6.7)$ & $18(17.0)$ & $6(17.1)$ & \\
\hline \multicolumn{5}{|l|}{ Severity of Illness Index (\%) } \\
\hline Level 1 & $1(1.7)$ & $1(0.9)$ & $0(0.0)$ & \multirow[t]{3}{*}{0.095} \\
\hline Level 2 & $50(83.3)$ & $79(74.5)$ & $21(60.0)$ & \\
\hline Level 3 & $9(15.0)$ & $26(24.5)$ & $14(40.0)$ & \\
\hline \multicolumn{5}{|l|}{ Activities of daily living } \\
\hline Median Katz index (median, IQR) & $6(6-6)$ & $1(0-3)$ & $0(0-0)$ & $<0.001$ \\
\hline \multicolumn{5}{|l|}{ Dependency in: } \\
\hline Bathing (\%) & $0(0.0)$ & $89(84.0)$ & $35(100)$ & $<0.001$ \\
\hline Toileting $(\%)$ & $0(0.0)$ & $83(78.3)$ & $35(100)$ & $<0.001$ \\
\hline Transfer $(\%)$ & $0(0.0)$ & $83(78.3)$ & $35(100)$ & $<0.001$ \\
\hline Dressing (\%) & $0(0.0)$ & $81(76.4)$ & $35(100)$ & $<0.001$ \\
\hline Continence $(\%)$ & $1(1.7)$ & $69(65.1)$ & $35(100)$ & $<0.001$ \\
\hline Feeding $(\%)$ & $0(0.0)$ & $40(37.7)$ & $35(100)$ & $<0.001$ \\
\hline \multicolumn{5}{|l|}{ Cognitive function } \\
\hline AMT (median, IQR) & $6(4-8)$ & $3(0-7)$ & $0(0-0)$ & $<0.001$ \\
\hline Dementia $(\%)$ & $12(20.0)$ & $54(50.9)$ & $28(80.0)$ & $<0.001$ \\
\hline Delirium on admission (\%) & $6(10.0)$ & $25(23.6)$ & $11(31.4)$ & 0.028 \\
\hline \multicolumn{5}{|l|}{ Admitting diagnosis } \\
\hline Sepsis (\%) & $34(56.7)$ & $62(58.5)$ & $22(62.9)$ & 0.838 \\
\hline Fall/syncope/seizure (\%) & $11(18.3)$ & $15(14.2)$ & $2(5.7)$ & 0.229 \\
\hline Delirium/dementia (\%) & $1(1.7)$ & $5(4.7)$ & $4(11.4)$ & 0.106 \\
\hline Other medical (\%) & $14(23.3)$ & $21(19.8)$ & $4(11.4)$ & 0.363 \\
\hline Surgical (\%) & $0(0.0)$ & $3(2.8)$ & $3(8.6)$ & 0.060 \\
\hline
\end{tabular}

$A M T$ abbreviate mental test, $C C I$ Charlson's comorbidity index, $C F S$ clinical frailty scale, $I Q R$ interquartile range, $S D$ standard deviation
$13.6 \%, p=0.01)$ and 12 -month (42.5\% vs. $21.2 \%, p=0.02)$ follow-up (Table 2). We also found that median total KI at baseline was significantly higher in surviving patients at 6 months [KI $1(0-3)$ vs. $1(0-1), p=0.02]$ and 12 months [KI $2(0-3)$ vs. $1(0-1), p=0.036)$. When CFS 6 was further categorised into KI 0-1 $(n=61)$ and KI 2-6 $(n=45)$, patients in the former group had a higher incidence of allcause mortality at 6 months $(31.1 \%$ vs. $8.9 \%, p=0.008)$ and 12 months $(39.3 \%$ vs. $15.6 \%, p=0.01)$ (Table 3 ).

In logistic regression analysis adjusted for age, gender, comorbidities, and severity of illness, we observed that feeding dependent was independently associated with mortality at 6 months (Odds Ratio (OR) 3.13, 95\% Confidence Interval (CI) 1.18-8.30, $p=0.022$ ) and 12 months (OR 2.66, 95\% CI 1.06-6.68, $p=0.038$ ) (Appendix 2). We also observed independent associations between dependency in bathing (OR 8.69, 95\% CI 1.05-71.74, $p=0.045$ ) and dressing (OR 3.87 (95\% CI 1.01-14.84), $p=0.049$ ) with 6-month institutionalisation.

\section{Predictive performances of mCFS-1 and mCFS-2}

We compared CFS against mCFS-1 and mCFS-2 in predicting incident mortality and institutionalisation. In univariate 
Table 2 Association between individual Katz Index items and negative health outcomes among CFS 6 patients

\begin{tabular}{|c|c|c|c|c|c|c|c|}
\hline \multirow{2}{*}{\multicolumn{2}{|c|}{$\begin{array}{l}\text { Premorbid Katz } \\
\text { Index (independ- } \\
\text { ent) }\end{array}$}} & \multicolumn{3}{|l|}{ Mortality } & \multicolumn{3}{|c|}{ Institutionalisation or mortality ${ }^{\mathrm{a}}$} \\
\hline & & $\begin{array}{l}\text { Initial hos- } \\
\text { pitalisation } \\
(n=106)\end{array}$ & 6 months $(n=106)$ & 12 months $(n=106)$ & $\begin{array}{l}\text { Initial hospitali- } \\
\text { sation }(n=106)\end{array}$ & 6 months $(n=105)^{\mathrm{b}}$ & 12 months $(n=105)^{\mathrm{b}}$ \\
\hline \multirow[t]{2}{*}{ Feeding } & Yes & $2 / 66(3.0 \%)$ & $9 / 66(13.6 \%)^{*}$ & $14 / 66(21.2 \%)^{*}$ & $6 / 66(9.1 \%)$ & $14 / 65(21.5 \%)$ & $21 / 65(32.3 \%)$ \\
\hline & No & $3 / 40(7.5 \%)$ & $14 / 40(35.0 \%)^{*}$ & $17 / 40(42.5 \%)^{*}$ & $4 / 40(10.0 \%)$ & $15 / 40(37.5 \%)$ & $18 / 40(45.0 \%)$ \\
\hline \multirow[t]{2}{*}{ Continence } & Yes & $2 / 37(5.4 \%)$ & $6 / 37(16.2 \%)$ & $8 / 37(21.6 \%)$ & $5 / 37(13.5 \%)$ & $10 / 36(27.8 \%)$ & $12 / 36(33.3 \%)$ \\
\hline & No & $3 / 69(4.3 \%)$ & $17 / 69(24.6 \%)$ & $23 / 69(33.3 \%)$ & $5 / 69(7.2 \%)$ & $19 / 69(27.5 \%)$ & $27 / 69(39.1 \%)$ \\
\hline \multirow[t]{2}{*}{ Dressing } & Yes & $0 / 25(0.0 \%)$ & $2 / 25(8.0 \%)$ & $5 / 25(20.0 \%)$ & $1 / 25(4.0 \%)$ & $3 / 24(12.5 \%)$ & $7 / 24(29.2 \%)$ \\
\hline & No & $5 / 81(6.2 \%)$ & $21 / 81(25.9 \%)$ & $26 / 81(32.1 \%)$ & 9/81 (11.1\%) & $26 / 81(32.1 \%)$ & $32 / 81(39.5 \%)$ \\
\hline \multirow[t]{2}{*}{ Transfer } & Yes & $1 / 23(4.3 \%)$ & $3 / 23(13.0 \%)$ & $5 / 23(21.7 \%)$ & $3 / 23(13.0 \%)$ & $5 / 22(22.7 \%)$ & $8 / 22(36.4 \%)$ \\
\hline & No & $4 / 83(4.8 \%)$ & $20 / 83(24.1 \%)$ & $26 / 83(31.3 \%)$ & $7 / 83(8.4 \%)$ & $24 / 83(28.9 \%)$ & $31 / 83(37.3 \%)$ \\
\hline \multirow[t]{2}{*}{ Toileting } & Yes & $0 / 23(0.0 \%)$ & $3 / 23(13.0 \%)$ & $5 / 23(21.7 \%)$ & $2 / 23(8.7 \%)$ & $5 / 22(22.7 \%)$ & $8 / 22(36.4 \%)$ \\
\hline & No & $5 / 83(6.0 \%)$ & $20 / 83(24.1 \%)$ & $26 / 83(31.3 \%)$ & $8 / 83(96 \%)$ & $24 / 83(28.9 \%)$ & $31 / 83(37.4 \%)$ \\
\hline \multirow[t]{2}{*}{ Bathing } & Yes & $0 / 17(0.0 \%)$ & $1 / 17(5.9 \%)$ & $3 / 17(17.6 \%)$ & $0 / 17(0.0 \%)$ & $1 / 17(5.9 \%)^{*}$ & $4 / 17(23.5 \%)$ \\
\hline & No & $5 / 89(5.6 \%)$ & $22 / 89(24.7 \%)$ & $28 / 89(31.5 \%)$ & $10 / 89(11.2 \%)$ & $28 / 88(31.8 \%)^{*}$ & $35 / 88(39.8 \%)$ \\
\hline
\end{tabular}

$C F S$ clinical frailty scale, $I Q R$ interquartile range, $S D$ standard deviation ${ }^{*} p<0.05$

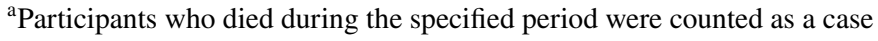

${ }^{\mathrm{b}} 1$ patient excluded from 6- and 12-month analyses due to loss to follow-up

Table 3 Association between Katz Index scores and negative health outcomes among CFS 6 patients

\begin{tabular}{|c|c|c|c|c|c|c|c|}
\hline \multicolumn{2}{|c|}{ Premorbid Katz Index } & \multicolumn{3}{|l|}{ Mortality } & \multicolumn{3}{|c|}{ Institutionalisation or mortality ${ }^{a}$} \\
\hline & & $\begin{array}{l}\text { Initial hospitali- } \\
\text { sation }(n=106)\end{array}$ & 6 months $(n=106)$ & $\begin{array}{l}12 \text { months } \\
(n=106)\end{array}$ & $\begin{array}{l}\text { Initial hospitali- } \\
\text { sation }(n=106)\end{array}$ & 6 months $(n=105)^{\mathrm{b}}$ & $\begin{array}{l}12 \text { months } \\
(n=105)^{\mathrm{b}}\end{array}$ \\
\hline \multirow[t]{7}{*}{ Total score } & 0 & $2 / 35(5.7 \%)$ & $11 / 35(31.4 \%)$ & $14 / 35(40.0 \%)$ & $3 / 35(8.6 \%)$ & $12 / 35(34.3 \%)$ & $15 / 35(42.9 \%)$ \\
\hline & 1 & $2 / 26(7.7 \%)$ & $8 / 26(30.8 \%)$ & $10 / 26(38.5 \%)$ & $2 / 26(7.7 \%)$ & $8 / 26(30.8 \%)$ & $11 / 26(42.3 \%)$ \\
\hline & 2 & $0 / 14(0.0 \%)$ & $0 / 14(0.0 \%)$ & $0 / 14(0.0 \%)$ & $2 / 14(14.3 \%)$ & $3 / 14(21.4 \%)$ & $3 / 14(21.4 \%)$ \\
\hline & 3 & $1 / 8(12.5 \%)$ & $1 / 8(12.5 \%)$ & $2 / 8(25.0 \%)$ & $2 / 8(25.0 \%)$ & $2 / 8(25.0 \%)$ & $3 / 8(37.5 \%)$ \\
\hline & 4 & $0 / 6(0.0 \%)$ & $2 / 6(33.3 \%)$ & $2 / 6(33.3 \%)$ & $0 / 6(0.0 \%)$ & $2 / 6(33.3 \%)$ & $2 / 6(33.3 \%)$ \\
\hline & 5 & $0 / 13(0.0 \%)$ & $1 / 13(7.7 \%)$ & $2 / 13(15.4 \%)$ & $1 / 13(7.7 \%)$ & $2 / 12(16.7 \%)$ & $4 / 12(33.3 \%)$ \\
\hline & 6 & $0 / 4(0.0 \%)$ & $0 / 4(0.0 \%)$ & $1 / 4(25.0 \%)$ & $0 / 4(0.0 \%)$ & $0 / 4(0.0 \%)$ & $1 / 4(25.0 \%)$ \\
\hline \multirow[t]{2}{*}{ Grouped by score } & $\begin{array}{l}\text { Katz } \\
0-1\end{array}$ & $4 / 61(6.6 \%)$ & $19 / 61(31.1 \%)^{*}$ & $24 / 61(39.3 \%)^{*}$ & $5 / 61(8.2 \%)$ & $20 / 61(32.8 \%)$ & $26 / 61(42.6 \%)$ \\
\hline & $\begin{array}{l}\text { Katz } \\
2-6\end{array}$ & $1 / 45(2.2 \%)$ & $4 / 45(8.9 \%) *$ & $7 / 45(15.6 \%)^{*}$ & $5 / 45(11.1 \%)$ & $9 / 44(20.5 \%)$ & $13 / 44(29.5 \%)$ \\
\hline
\end{tabular}

$C F S$ clinical frailty scale, $I Q R$ interquartile range, $S D$ standard deviation ${ }^{*} p<0.05$

a Participants who died during the specified period were counted as a case

${ }^{\mathrm{b}} 1$ patient excluded from 6- and 12-month analyses due to loss to follow-up

analysis, all three tools showed significant association with mortality at 6 and 12 months (both $p<0.001$ ) and with institutionalisation at initial hospitalisation $(p<0.05)$, 6 months $(p<0.001)$ and 12 months $(p<0.001)$. For both mCFS- 1 and mCFS-2, there was an increase in mortality and institutionalisation moving from CFS 5 through CFS 6 stages to CFS 7-8 at 6- and 12-month follow-up (Table 4).

We next performed logistic regression analysis, adjusting for age, gender, comorbidities, and severity of illness (Table 5). When taken as a whole, CFS 6 had a fourfold 
Table 4 Comparison between CFS, mCFS-1, and mCFS-2 against incident mortality and institutionalisation

\begin{tabular}{|c|c|c|c|c|c|c|c|}
\hline \multirow[t]{2}{*}{ Frailty category } & & \multicolumn{3}{|l|}{ Mortality } & \multicolumn{3}{|c|}{ Institutionalisation or mortality ${ }^{\mathrm{a}}$} \\
\hline & & $\begin{array}{l}\text { Initial hospitalisa- } \\
\text { tion }(n=201)(\%)\end{array}$ & $\begin{array}{l}6 \text { months }(n=201) \\
(\%)\end{array}$ & $\begin{array}{l}12 \text { months } \\
(n=201)(\%)\end{array}$ & $\begin{array}{l}\text { Initial hospitalisa- } \\
\text { tion }(n=201)(\%)\end{array}$ & $\begin{array}{l}\text { 6-month } \\
(n=197)^{\mathrm{b}}(\%)\end{array}$ & $\begin{array}{l}\text { 12-month } \\
(n=197)^{\mathrm{b}}(\%)\end{array}$ \\
\hline \multirow{3}{*}{$\begin{array}{l}\text { Clinical Frailty } \\
\text { Scale (CFS) }\end{array}$} & 5 & $0 / 60(0.0)$ & $3 / 60(5.0)^{\dagger}$ & $5 / 60(8.3)^{\dagger}$ & $0 / 60(0.0)^{*}$ & $4 / 57(7.0)^{\dagger}$ & $6 / 57(10.5)^{\dagger}$ \\
\hline & 6 & $5 / 106(4.7)$ & $23 / 106(21.7)^{\dagger}$ & $31 / 106(29.2)^{\dagger}$ & $10 / 106(9.4)^{*}$ & $29 / 105(27.6)^{\dagger}$ & $39 / 105(37.1)^{\dagger}$ \\
\hline & $7-8$ & $3 / 35(8.6)$ & $16 / 35(45.7)^{\dagger}$ & $21 / 35(60.0)^{\dagger}$ & $3 / 35(8.6)^{*}$ & $18 / 35(51.4)^{\dagger}$ & $22 / 35(62.9)^{\dagger}$ \\
\hline \multirow{4}{*}{$\begin{array}{c}\text { Modified CFS } \\
\text { Version } 1 \\
(\mathrm{mCFS}-1)\end{array}$} & 5 & $0 / 60(0.0)$ & $3 / 60(5.0)^{\dagger}$ & $5 / 60(8.3)^{\dagger}$ & $0 / 60(0.0)^{*}$ & $4 / 57(7.0)^{\dagger}$ & $6 / 57(10.5)^{\dagger}$ \\
\hline & $6 \mathrm{~A}$ & $1 / 45(2.2)$ & $4 / 45(8.9)^{\dagger}$ & $7 / 45(15.6)^{\dagger}$ & $5 / 45(11.1)^{*}$ & $9 / 44(20.5)^{\dagger}$ & $13 / 44(29.5)^{\dagger}$ \\
\hline & $6 \mathrm{~B}$ & $4 / 61(6.6)$ & $19 / 61(31.1)^{\dagger}$ & $24 / 61(39.3)^{\dagger}$ & $5 / 61(8.2)^{*}$ & $20 / 61(32.8)^{\dagger}$ & $26 / 61(42.6)^{\dagger}$ \\
\hline & $7-8$ & $3 / 35(8.6)$ & $16 / 35(45.7)^{\dagger}$ & $21 / 35(60.0)^{\dagger}$ & $3 / 35(8.6)^{*}$ & $18 / 35(51.4)^{\dagger}$ & $22 / 35(62.9)^{\dagger}$ \\
\hline \multirow{5}{*}{$\begin{array}{l}\text { Modified CFS } \\
\text { Version } 2 \\
(\mathrm{mCFS}-2)\end{array}$} & 5 & $0 / 60(0.0)$ & $3 / 60(5.0)^{\dagger}$ & $5 / 60(8.3)^{\dagger}$ & $0 / 60(0.0)^{*}$ & $4 / 57(7.0)^{\dagger}$ & $6 / 57(10.5)^{\dagger}$ \\
\hline & $6 \mathrm{~A}$ & $1 / 45(2.2)$ & $4 / 45(8.9)^{\dagger}$ & $7 / 45(15.6)^{\dagger}$ & $5 / 45(11.1)^{*}$ & $9 / 44(20.5)^{\dagger}$ & $13 / 44(29.5)^{\dagger}$ \\
\hline & $6 \mathrm{~B} 1$ & $1 / 21(4.8)$ & $5 / 21(23.8)^{\dagger}$ & $7 / 21(33.3)^{\dagger}$ & $1 / 21(4.8)^{*}$ & $5 / 21(23.8)^{\dagger}$ & $8 / 21(38.1)^{\dagger}$ \\
\hline & $6 \mathrm{~B} 2$ & $3 / 40(7.5)$ & $14 / 40(35.0)^{\dagger}$ & $17 / 40(42.5)^{\dagger}$ & $4 / 40(10.0)^{*}$ & $15 / 40(37.5)^{\dagger}$ & $18 / 40(45.0)^{\dagger}$ \\
\hline & $7-8$ & $3 / 35(8.6)$ & $16 / 35(45.7)^{\dagger}$ & $21 / 35(60.0)^{\dagger}$ & $3 / 35(8.6)^{*}$ & $18 / 35(51.4)^{\dagger}$ & $22 / 35(62.9)^{\dagger}$ \\
\hline
\end{tabular}

$* p<0.05,{ }^{\dagger} p<0.001$ (Fisher Exact test)

${ }^{\text {a }}$ Participants who died during the specified period were counted as a case

b4 patients excluded from 6- and 12-month analyses due to loss to follow-up

increase in mortality at 6 and 12 months compared to CFS 5. When CFS 6 was stratified into subcategories, CFS 6A did not increase mortality at 6 and 12 months compared with CFS 5 (OR 1.64 and 1.83, respectively), unlike CFS 6B (OR 7.13 and 6.33 , respectively) in mCFS-1. Further delineation of CFS 6B in mCFS-2 revealed differences in mortality in 6B1 (OR 5.16 and 5.19, respectively) and 6B2 (OR 8.18 and 6.92. respectively). Similarly, CFS 6 increased institutionalisation by around fivefold at 6 and 12 months. In mCFS-1 model, CFS 6B significantly increased institutionalisation (OR 5.85 and 6.16, respectively) more than CFS 6A (OR 3.27 and 3.55, respectively). Again, further categorisation of CFS 6B in mCFS-2 revealed differences in institutionalisation in 6B1 (OR 3.89 and 5.44, respectively) and 6B2 (OR 6.99 and 6.55, respectively). Our results were similar when we ran a separate regression model substituting comorbidities with underlying dementia when adjusting for cofounders.

Lastly, AUC for CFS, mCFS- 1 and mCFS- 2 against incident mortality at 12 months was $0.72(95 \%$ CI $0.65-0.78$, $p<0.001), 0.76$ (95\% CI 0.69-0.81, $p<0.001$ ), and 0.76 (95\% CI $0.70-0.82, p<0.001$ ), respectively (Table 6). Optimal cut-point was 6 for CFS (sensitivity $91.2 \%$, specificity $38.2 \%$ ) and $6 \mathrm{~B}$ for either mCFS-1 or mCFS-2 (sensitivity $79.0 \%$, specificity $64.6 \%$ ). Fairly similar AUC and optimal cut-point results were noted for institutionalisation at 12 months. However, results for institutionalisation at initial hospitalisation and 6 months were largely inconsistent.

\section{Discussion}

In this retrospective cohort study of hospitalised oldest old adults, we found that moderately frail (CFS 6) patients have greater heterogeneity in functional status compared to other frail categories. This in turn resulted in a wider range of risk estimates for adverse health outcomes of mortality and institutionalisation at 6 and 12 months, such that patients with $\mathrm{KI} \geq 2$ have lower mortality risk which approximate that of CFS 5, whereas those with $\mathrm{KI} \leq 1$ and feeding dependent are at greatest risk. Incorporating CFS 6 subcategories based on bADL functional status increased predictive performance for longitudinal adverse outcomes compared with the original CFS scoring. To our knowledge, this is the first study to report these findings.

Findings in this study are consistent with an earlier study which reported that disability in bADLs is a strong predictor of survival amongst centenarians [20]. A recent study reported an adjusted hazard ratio per disability increment in KI of 1.6 among hospitalised patients aged 83 years and above [21]. Notably, an ordered hierarchy of ADL has been previously reported, with feeding being the easiest and bathing the most difficult [22]. Thus, loss of independence in feeding represents a more advanced state of bADL functional dependency which would portend poorer outcomes. A study of 418 older adults who underwent videofluoroscopic swallow for dysphagia reported that malnutrition and frailty were positively correlated with severe dysphagia, 
Table 5 Multivariate analyses comparing CFS, mCFS-1, and mCFS-2 against incident mortality and institutionalisation

\begin{tabular}{|c|c|c|c|c|c|c|c|}
\hline \multirow[t]{3}{*}{ Frailty category } & & \multicolumn{3}{|l|}{ Mortality } & \multicolumn{3}{|c|}{ Institutionalisation or mortality $^{\mathrm{b}}$} \\
\hline & & $\begin{array}{l}\text { Initial hospitalisa- } \\
\text { tion }(n=201)\end{array}$ & $\begin{array}{l}6 \text { months } \\
(n=201)\end{array}$ & $\begin{array}{l}12 \text { months } \\
(n=201)\end{array}$ & $\begin{array}{l}\text { Initial hospitalisa- } \\
\text { tion }(n=201)\end{array}$ & $\begin{array}{l}6 \text { months } \\
(n=197)^{\mathrm{c}}\end{array}$ & $\begin{array}{l}12 \text { months } \\
(n=197)^{\mathrm{c}}\end{array}$ \\
\hline & & \multicolumn{3}{|c|}{ Adjusted OR (95\% CI) } & \multicolumn{3}{|c|}{ Adjusted OR (95\% CI) } \\
\hline \multirow{4}{*}{$\begin{array}{l}\text { Clinical Frailty } \\
\text { Scale (CFS) }\end{array}$} & 5 & $--^{\mathrm{a}}$ & Ref & Ref & $--^{\mathrm{a}}$ & Ref & Ref \\
\hline & 6 & Ref & $\begin{array}{l}4.30(1.20- \\
15.46)^{*}\end{array}$ & $\begin{array}{l}3.90(1.36- \\
11.16)^{*}\end{array}$ & Ref & $\begin{array}{c}4.59(1.49- \\
14.11)^{*}\end{array}$ & $4.83(1.84-12.67)^{*}$ \\
\hline & $7-8$ & $2.16(0.46-10.14)$ & $\begin{array}{l}14.08(3.56- \\
55.80)^{\dagger}\end{array}$ & $\begin{array}{l}16.35(4.85- \\
55.10)^{\dagger}\end{array}$ & $1.05(0.26-4.27)$ & $\begin{array}{l}13.23(3.80- \\
46.05)^{\dagger}\end{array}$ & $\begin{array}{l}15.16(4.80- \\
47.91)^{\dagger}\end{array}$ \\
\hline & & \multicolumn{3}{|c|}{ Adjusted OR (95\% CI) } & \multicolumn{3}{|c|}{ Adjusted OR (95\% CI) } \\
\hline \multirow{3}{*}{$\begin{array}{l}\text { Modified CFS } \\
\text { Version } 1 \\
\text { (mCFS-1) }\end{array}$} & 5 & $-^{\mathrm{a}}$ & Ref & Ref & $-^{\mathrm{a}}$ & Ref & Ref \\
\hline & $\begin{array}{l}6 \mathrm{~A} \\
6 \mathrm{~B} \\
7-8\end{array}$ & $\begin{array}{l}\text { Ref } \\
2.39(0.24-23.93) \\
3.97(0.38-42.12)\end{array}$ & $\begin{array}{l}1.64(0.34-7.92) \\
7.13(1.90- \\
26.83)^{*} \\
14.57(3.66- \\
57.96)^{\dagger}\end{array}$ & $\begin{array}{l}1.83(0.52-6.48) \\
6.33(2.07- \\
19.33)^{*} \\
17.03(5.02- \\
57.84)^{\dagger}\end{array}$ & $\begin{array}{l}\text { Ref } \\
0.68(0.17-2.77) \\
0.85(0.18-4.14)\end{array}$ & $\begin{array}{l}3.27(0.92-11.57) \\
5.85(1.79- \\
19.10)^{*} \\
13.47(3.86- \\
46.97)^{\dagger}\end{array}$ & $\begin{array}{l}3.55(1.19-10.52)^{*} \\
6.16(2.19-17.38)^{*} \\
15.51(4.89- \\
49.19)^{\dagger}\end{array}$ \\
\hline & & \multicolumn{3}{|c|}{ Adjusted OR (95\% CI) } & \multicolumn{3}{|c|}{ Adjusted OR (95\% CI) } \\
\hline \multirow{5}{*}{$\begin{array}{l}\text { Modified CFS } \\
\text { Version } 2 \\
\text { (mCFS-2) }\end{array}$} & 5 & $--^{\mathrm{a}}$ & Ref & Ref & $-^{\mathrm{a}}$ & Ref & Ref \\
\hline & $6 \mathrm{~A}$ & Ref & $1.64(0.34-7.91)$ & $1.83(0.52-6.47)$ & Ref & $3.26(0.92-11.55)$ & $3.54(1.19-10.51)^{*}$ \\
\hline & $6 \mathrm{~B} 1$ & $1.54(0.09-27.81)$ & $\begin{array}{l}5.16(1.05- \\
25.50)^{*}\end{array}$ & $\begin{array}{l}5.19(1.30- \\
20.69)^{*}\end{array}$ & $0.33(0.03-3.17)$ & $3.89(0.89-16.92)$ & $5.44(1.51-19.57)^{*}$ \\
\hline & $6 \mathrm{~B} 2$ & $2.94(0.27-32.17)$ & $\begin{array}{l}8.18(2.08- \\
32.18)^{*}\end{array}$ & $\begin{array}{l}6.92(2.14- \\
22.35)^{*}\end{array}$ & $0.95(0.21-4.25)$ & $\begin{array}{l}6.99(2.04- \\
23.98)^{*}\end{array}$ & $6.55(2.19-19.63)^{*}$ \\
\hline & $7-8$ & $3.98(0.38-42.37)$ & $\begin{array}{l}14.46(3.64- \\
57.42)^{\dagger}\end{array}$ & $\begin{array}{l}16.93(4.99- \\
57.44)^{\dagger}\end{array}$ & $0.86(0.17-4.20)$ & $\begin{array}{c}13.39(3.84- \\
46.64)^{\dagger}\end{array}$ & $\begin{array}{l}15.47(4.88- \\
49.03)^{\dagger}\end{array}$ \\
\hline
\end{tabular}

CFS Clinical Frailty Scale, $C I$ confidence interval, $O R$ odds ratio, Ref reference

Adjusted for age, gender, comorbidities, and severity of illness

${ }^{a}$ No case of mortality or institutionalisation in CFS category 5 at initial hospitalization

$* p<0.05$

${ }^{\dagger} p<0.001$

${ }^{\mathrm{b}}$ Participants who died during the specified period were counted as a case

${ }^{c} 4$ patients excluded from 6- and 12q-month analyses due to loss to follow-up

irrespective of age [23]. Additionally, dependency in feeding has been shown to be a powerful predictor of poorer outcomes including mortality [24]. Our findings, along with the aforementioned studies, support the utility of supplementing $\mathrm{KI}$ and feeding dependency into the CFS matrix to improve its predictive performance.

Earlier studies reported that amongst older adults who are hospitalised or admitted to the intensive care unit, the mildly frail (CFS 5) had better outcomes than the moderately or severely frail, even in the oldest old $[25,26]$. Our study adds to this body of evidence by demonstrating that CFS 6 represents a heterogeneous group with different prognosis depending on the underlying bADL functional status. Specifically, moderately frail hospitalised older adults with better functional abilities (categorised as CFS $6 \mathrm{~A}$ in mCFS-1 or mCFS-2) may potentially benefit from more aggressive interventions, including critical care, as they appear to share similar survival outcomes with CFS 5 patients up to 12-month follow-up. In contrast, in moderately frail hospitalised older adults with impairment across bADLs (categorised as CFS 6B), feeding dependency can further differentiate the prognosis, such that those who are feeding independent (categorised as CFS 6B1) have around one-third the mortality and institutionalisation risks compared with CFS 7, vis-à-vis one-half the risks in those who are feeding dependent (categorised as CFS 6B2).

Our study findings are salient in light of the current COVID-19 pandemic, when there is intense interest in reliable assessment tools to inform patient prioritisation for scarce intensive care resource. A recent multicentre study of patients admitted to hospital with COVID-19 found that frailty better predicted mortality compared to age or comorbidity [27]. Yet, findings from a single-site study of hospitalised patients aged 70 and above suggest that frailty was 
Table 6 AUC comparing CFS, mCFS-1 and mCFS-2 in predicting incident mortality and institutionalisation

\begin{tabular}{|c|c|c|c|c|c|c|}
\hline \multirow[t]{2}{*}{ Adverse outcomes } & \multicolumn{2}{|c|}{ Initial hospitalisation } & \multicolumn{2}{|l|}{6 months } & \multicolumn{2}{|l|}{12 months } \\
\hline & $\operatorname{AUC}(95 \% \mathrm{CI})$ & $\begin{array}{l}\text { Ideal cut off cat- } \\
\text { egory }(\mathrm{Sn} \%, \mathrm{Sp} \%)\end{array}$ & $\mathrm{AUC}(95 \% \mathrm{CI})$ & $\begin{array}{l}\text { Ideal cut off cat- } \\
\text { egory }(\mathrm{Sn} \%, \mathrm{Sp} \%)\end{array}$ & $\operatorname{AUC}(95 \% \mathrm{CI})$ & $\begin{array}{l}\text { Ideal cut off } \\
\text { category }(\mathrm{Sn} \% \text {, } \\
\text { Sp\%) }\end{array}$ \\
\hline \multicolumn{7}{|l|}{ Mortality } \\
\hline CFS & $0.70(0.63-0.76)^{*}$ & $\geq 6(100,31.1)$ & $0.71(0.64-0.77)^{\dagger}$ & $\geq 6(92.9,35.9)$ & $0.72(0.65-0.78)^{\dagger}$ & $\geq 6(91.2,38.2)$ \\
\hline mCFS-1 & $0.74(0.67-0.80)^{\dagger}$ & $\geq 6 \mathrm{~B}(87.5,53.9)$ & $0.76(0.69-0.81)^{\dagger}$ & $\geq 6 \mathrm{~B}(83.3,61.6)$ & $0.76(0.69-0.81)^{\dagger}$ & $\geq 6 \mathrm{~B}(79.0,64.6)$ \\
\hline mCFS-2 & $0.75(0.68-0.81)^{\dagger}$ & $\geq 6 \mathrm{~B} 1(87.5,53.9)$ & $0.76(0.70-0.82)^{\dagger}$ & $\geq 6 \mathrm{~B} 1(83.3,61.6)$ & $0.76(0.70-0.82)^{\dagger}$ & $\geq 6 \mathrm{~B} 1(79.0,64.6)$ \\
\hline \multicolumn{7}{|l|}{$\begin{array}{l}\text { Institutionalisation } \\
\text { or mortality }{ }^{\mathrm{a}, \mathrm{b}}\end{array}$} \\
\hline CFS & $0.65(0.58-0.72)^{*}$ & $\geq 6(100,31.9)$ & $0.70(0.63-0.76)^{\dagger}$ & $\geq 6(92.2,36.3)$ & $0.71(0.64-0.77)^{\dagger}$ & $\geq 6(91.0,39.2)$ \\
\hline mCFS-1 & $0.64(0.57-0.70)^{*}$ & $\geq 6 \mathrm{~A}(100,31.9)$ & $0.72(0.66-0.78)^{\dagger}$ & $\geq 6 \mathrm{~B}(74.5,60.3)$ & $0.73(0.66-0.79)^{\dagger}$ & $\geq 6 \mathrm{~B}(71.6,63.1)$ \\
\hline mCFS-2 & $0.65(0.58-0.71)^{*}$ & $\geq 6 \mathrm{~A}(100,31.9)$ & $0.73(0.66-0.79)^{\dagger}$ & $\geq 6 \mathrm{~B} 2(64.7,71.2)$ & $0.73(0.66-0.79)^{\dagger}$ & $\geq 6 \mathrm{~B} 1(71.6,63.1)$ \\
\hline
\end{tabular}

$A U C$ area under the operator curve; CFS, clinical frailty scale; CI, confidence interval; Sn, sensitivity; Sp, specificity

$* p<0.05$

${ }^{\dagger} p<0.001$

aparticipants who died during the specified period were counted as a case

b4 patients excluded from 6- and 12-month analyses due to loss to follow-up

not a good discriminator of prognosis in COVID-19 [28]. Nonetheless, we advise that frailty is but one component of a multi-pronged person-centred approach to assist prognostication and to guide meaningful conversations on goals of care, i.e. to set goals of care rather than shift the goalpost of care $[29,30]$.

This study had a number of limitations. First, the exclusion of dangerously ill patients likely contributed to the overall low inpatient mortality rate, and limits generalisability of our study findings. Second, four participants were lost to follow-up and were excluded from 6- and 12-month analyses. Even so, we were able to determine their mortality status by accessing local hospital electronic medical records. Third, whilst it is reassuring that the modified CFS discriminated predictive performance in our population of hospitalised oldest old adults, future studies across the age range of older adults and in different study settings are required to ascertain the generalisability of our results. Fourth, our assessment of bADL was limited to the KI and we are unable to examine whether the use of other instruments such as the Barthel index would produce better results. Nevertheless, a study of 86 centenarians (mean age $102 \pm 1$ years) found that KI performed similarly to the Barthel index in predicting 360-day mortality [20]. Therefore, the ease of use of the KI is an advantage when used to derive a quick assessment of CFS score. And lastly, we acknowledge that despite the clear definitions in place to categorise patients as CFS 6 using the CFS-A, our data show that there remains great variability in function, with some CFS 6 participants having KI scores of
0 (fully dependent) or 6 (fully independent). This reflects the fact that there remains variability in scoring the CFS in actual practice, and hence, the added approach of including bADLs may help to address this concern.

In conclusion, this study corroborates the heterogeneity in bADL functional status with downstream impact on predictive performance of risk estimates of mortality and institutionalisation among hospitalised older adults with moderately frailty. Other indicators including a KI score of 1 or less and dependency in feeding should be taken into account to better prognosticate and aid clinical decisionmaking in moderately frail individuals. Taken together, our study validates the use of a modified approach in subgrouping CFS 6 via bADL functional status for improved predictive performance.

Acknowledgements The authors would like to thank the research team of Dr. Esther Ho, Dr. Jewel Baldevarona-Llego and Lynn Wu for their contributions in data collection. The authors would also like to express their gratitude to Dr. Laura Tay who contributed greatly to the initial concept and design of the study.

Author contributions All authors contributed to the study conception and design. Material preparation, data collection and analysis were performed by EC. All authors contributed to the interpretation of data. The first draft of the manuscript was written by EC and all authors commented on previous versions of the manuscript. All authors read and approved the final manuscript.

Funding This research did not receive any funding from agencies in the public, commercial, or not-for-profit sectors. 


\section{Compliance with ethical standards}

Conflict of interest The authors declare that they have no conflict of interest.

Ethics approval Ethics approval for the study was obtained from the National Healthcare Group (NHG) Domain Specific Review Board (DSRB). The procedures used in this study adhere to the tenets of the Declaration of Helsinki.

Consent to participate and publication Written consent was obtained from patients or a legally acceptable representative (if patients lack mental capacity for consenting) for participation in the study and publication of data.

\section{References}

1. Morley JE, Vellas B, van Kan GA et al (2013) Frailty consensus: a call to action. J Am Med Dir Assoc 14(6):392-397. https://doi. org/10.1016/j.jamda.2013.03.022

2. Woo $\mathbf{J}$ (2018) Challenges of population ageing: putting frailty as a cornerstone of health and social care systems. Eur Geriatr Med 9:273-276. https://doi.org/10.1007/s41999-018-0056-0

3. Dent E, Morley JE, Cruz-Jentoft AJ et al (2019) Physical Frailty: ICFSR international clinical practice guidelines for identification and management. J Nutr Health Aging 23(9):771-787. https://doi. org/10.1007/s12603-019-1273-z

4. Rockwood K, Song X, MacKnight C et al (2005) A global clinical measure of fitness and frailty in elderly people. CMAJ 173(5):489-495. https://doi.org/10.1503/cmaj.050051

5. Chong E, Ho E, Baldevarona-Llego J, Chan M, Wu L, Tay L (2017) Frailty and risk of adverse outcomes in hospitalized older adults: a comparison of different frailty measures. J Am Med Dir Assoc 18(7):638.e7-638.e11. https://doi.org/10.1016/j.jamda .2017 .04 .011

6. Chong E, Ho E, Baldevarona-Llego J et al (2018) Frailty in hospitalized older adults: comparing different frailty measures in predicting short- and long-term patient outcomes. J Am Med Dir Assoc 19(5):450-457.e3. https://doi.org/10.1016/j.jamda .2017.10.006

7. Chong E, Chia JQ, Law F, Chew J, Chan M, Lim WS (2019) Validating a standardised approach in administration of the clinical frailty scale in hospitalised older adults. Ann Acad Med Singap 48(4):115-124

8. Chu L, Shi C (2020) Application of CSHA Frailty Index and clinical frailty scale in geriatric assessment of elderly males in China. Am J Nurs Sci 9(4):240-243. https://doi.org/10.11648 /j.ajns.20200904.22

9. Dent E, Lien C, Lim WS et al (2017) The Asia-Pacific clinical practice guidelines for the management of frailty [published correction appears in J Am Med Dir Assoc. 2018 Jan;19(1):94]. J Am Med Dir Assoc 18(7):564-575. https://doi.org/10.1016/j.jamda .2017.04.018

10. Juma S, Taabazuing M-M, Montero-Odasso M (2016) Clinical Frailty Scale in an acute medicine unit: a simple tool that predicts length of stay. Can Geriatr J 19(2):34-39. https://doi.org/10.5770/ cgj.19.196

11. Hatcher VH, Galet C, Lilienthal M, Skeete DA, Romanowski KS (2019) Association of Clinical Frailty Scores with hospital readmission for falls after index admission for trauma-related injury. JAMA Netw Open. 2(10):e1912409. https://doi.org/10.1001/ jamanetworkopen.2019.12409 (Published 2019 Oct 2)
12. Darvall JN, Loth J, Bose T et al (2020) Accuracy of the Clinical Frailty Scale for perioperative frailty screening: a prospective observational study. Can J Anaesth 67(6):694-705. https://doi. org/10.1007/s12630-020-01610-x

13. Chan DC, Tsou HH, Chen CY, Chen CY (2010) Validation of the Chinese-Canadian study of health and aging clinical frailty scale (CSHA-CFS) telephone version. Arch Gerontol Geriatr 50(3):e74-e80. https://doi.org/10.1016/j.archger.2009.06.004

14. Davies J, Whitlock J, Gutmanis I, Kane SL (2018) Inter-rater reliability of the retrospectively assigned clinical frailty scale score in a geriatric outreach population. Can Geriatr J 21(1):1-5. https ://doi.org/10.5770/cgj.21.263

15. Scarborough JE, Bennett KM, Englum BR, Pappas TN, LagooDeenadayalan SA (2015) The impact of functional dependency on outcomes after complex general and vascular surgery. Ann Surg 261(3):432-437. https://doi.org/10.1097/SLA.0000000000000767

16. Gullón A, Formiga F, Camafort $M$ et al (2018) Baseline functional status as the strongest predictor of in-hospital mortality in elderly patients with non-valvular atrial fibrillation: results of the NONAVASC registry. Eur J Intern Med 47:69-74. https://doi. org/10.1016/j.ejim.2017.09.020

17. Chin BS, Kim MS, Han SH et al (2011) Risk factors of all-cause in-hospital mortality among Korean elderly bacteremic urinary tract infection (UTI) patients. Arch Gerontol Geriatr 52(1):e50e55. https://doi.org/10.1016/j.archger.2010.05.011

18. Charlson ME, Pompei P, Ales KL, MacKenzie CR (1987) A new method of classifying prognostic comorbidity in longitudinal studies: development and validation. J Chronic Dis 40(5):373-383. https://doi.org/10.1016/0021-9681(87)90171-8

19. Wong WC, Sahadevan S, Ding YY et al (2010) Resource consumption in hospitalized, frail older patients. Ann Acad Med Singap 39:830-836

20. Szewieczek J, Francuz T, Dulawa J et al (2015) Functional measures, inflammatory markers and endothelin-1 as predictors of 360day survival in centenarians. Age (Dordrecht) 37(5):85. https:// doi.org/10.1007/s11357-015-9822-9

21. Ritt M, Ritt JI, Sieber CC, Gaßmann KG (2017) Comparing the predictive accuracy of frailty, comorbidity, and disability for mortality: a 1-year follow-up in patients hospitalized in geriatric wards. Clin Interv Aging. 12:293-304. https://doi.org/10.2147/ CIA.S124342 (Published 2017 Feb 8)

22. Gerrard P (2013) The hierarchy of the activities of daily living in the Katz index in residents of skilled nursing facilities. J Geriatr Phys Ther 36(2):87-91. https://doi.org/10.1519/JPT.0b013e3182 $68 \mathrm{da} 23$

23. Ahn DH, Yang HE, Kang HJ et al (2020) Changes in etiology and severity of dysphagia with aging. Eur Geriatr Med 11(1):139-145. https://doi.org/10.1007/s41999-019-00259-0

24. Siebens H, Trupe E, Siebens A et al (1986) Correlates and consequences of eating dependency in institutionalized elderly. J Am Geriatr Soc 34(3):192-198. https://doi. org/10.1111/j.1532-5415.1986.tb04202.x

25. Darvall JN, Bellomo R, Paul E et al (2019) Frailty in very old critically ill patients in Australia and New Zealand: a populationbased cohort study. Med J Aust 211(7):318-328

26. Chong E, Chan M, Tan HN, Lim WS (2020) COVID-19: use of the Clinical Frailty Scale for critical care decisions. J Am Geriatr Soc 68(6):E30-E32. https://doi.org/10.1111/jgs.16528

27. Hewitt J, Carter B, Vilches-Moraga A et al (2020) The effect of frailty on survival in patients with COVID-19 (COPE): a multicentre, European, observational cohort study. Lancet Public Health. https://doi.org/10.1016/S2468-2667(20)30146-8 (published online ahead of print, 2020 Jun 30)

28. Miles A, Webb TE, Mcloughlin BC et al (2020) Outcomes from COVID-19 across the range of frailty: excess mortality in fitter 
older people. Eur Geriatr Med. https://doi.org/10.1007/s4199 9-020-00354-7 (published online ahead of print, 2020 Jul 18)

29. Hubbard RE, Maier AB, Hilmer SN, Naganathan V, Etherton-Beer C, Rockwood K (2020) Frailty in the face of COVID-19. Age Ageing 49(4):499-500. https://doi.org/10.1093/ageing/afaa095

30. Hsien-Xiong Lee R, Peiying Ho E, Neo HY, Hum A, Lim WS (2020) Letter to the Editor: Setting Goals or Shifting Goalposts: Role of Frailty for Critical Care Decisions during COVID-19. J Frailty Aging. 9(4):246-247. https://doi.org/10.14283/jfa.2020.46
Publisher's Note Springer Nature remains neutral with regard to jurisdictional claims in published maps and institutional affiliations. 